\title{
Paper of the October Issue of Stem Cell Reviews and Reports Addresses a Role of Prenatal Exposure of Murine Testicular Stem Cells to Endocrine Disrupting Chemicals that Results in Defective Spermatogenesis, Reduced Fertility and Tumor Initiation in Adult Life
}

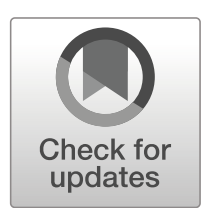

\author{
Mariusz Z. Ratajczak ${ }^{1}$ \\ (C) Springer Science+Business Media, LLC, part of Springer Nature 2020
}

This month we have selected the work of Dr. Anita Kaushik in the outstanding research group of Dr. Deepa Bhartiya, entitled "Altered Biology of Testicular VSELs and SSCs by Neonatal Endocrine Disruption Results in Defective Spermatogenesis, Reduced Fertility and Tumor Initiation in Adult Mice".

Endocrine-disrupting chemicals (EDCs) are present ubiquitously in the environment and pose a serious threat to global human health, affecting multiple organs including reproductive disorders and cancers. Perinatal exposure to the EDCs lead to diseases in adult life supporting the concept of fetal origin of adult disease. Reproductive health of men has declined in recent times as evidenced by decreased sperm count and increased incidence of infertility and testicular cancer. Young men are getting affected by testicular cancers. Scientists have been working worldwide to understand how EDCs threaten human health but there is no clear understanding as yet. In the current volume of SCRR, Kaushik et al. hypothesized that possibly the testicular stem cells are affected by EDCs during perinatal stage and being immortal in nature (survive throughout life unlike somatic cells with shorter life span) manifest disease state in adult life. Stem cells function in a subtle manner to maintain tissue homeostasis. A strict balance between self-renewal, expansion and differentiation of tissue stem cells results in maintaining tissue homeostasis and any perturbation to this balance will lead to disease state.

The group exposed mouse pups to endocrine disruption with estradiol (E2, $20 \mu \mathrm{g} / \mathrm{pup} /$ day on days 5-7) and diethylstilbestrol (DES, $2 \mu \mathrm{g} / \mathrm{pup} /$ day on days 1-5) and studied how the testicular stem cells are affected in 100 days old adult mice. Testes harbors two populations of stem cells including very small embryonic-like stem cells (VSELs) and spermatogonial stem cells (SSCs). In a sister paper they first discussed an interesting method to enrich testicular stem cells. After enzymatic digestion, cells suspension was first centrifuged at $1000 \mathrm{rpm}$ when majority of cells pellet down and later spinning the supernatant at $3000 \mathrm{rpm}$ resulted in almost ten-fold enrichment of the stem cells. Besides expressing pluripotent markers, OCT-4A positive VSELs expressed ER $\alpha, E R \beta$ and FSHR and thus are vulnerable to EDCs.

Neonatal endocrine disruption resulted in reduced sperm count, infertility and DES treatment further resulted in testicular cancer-like changes. Authors for the first time show that cancer-like changes in DES treated testes was associated with excessive self-renewal of VSELs (more than 6 folds increase in VSELs numbers by flow cytometry; $>10$ folds increased expression of OCT-4A and $>40$ folds increase in Nanog and Sox 2 by qRT-PCR). Blocked differentiation/spermatogenesis resulted in reduced sperm count \& infertility.

The authors concluded that EDCs affect tissue-resident stem cells biology to result in various diseases in adult life. VSELs maintain life-long tissue homeostasis and also initiate cancer. OCT-4A is the most sensitive and specific marker for testicular cancers.

Publisher's Note Springer Nature remains neutral with regard to jurisdictional claims in published maps and institutional affiliations.

Mariusz Z. Ratajczak

mariusz.ratajczak@louisville.edu

1 University of Louisville, Louisville, USA 